\title{
Microchannel structure of ceramic membranes for oxygen
}

\section{separation}

\author{
Xin Shao ${ }^{a}$, Zhitao Wang ${ }^{a}$, Shanshan Xu ${ }^{a, b}$, Kui Xie $^{c}$, Xun Hu ${ }^{a}$, Dehua Dong ${ }^{a}{ }^{*}$, Gordon Parkinson ${ }^{a}$, \\ Chun-Zhu Li ${ }^{a}$ \\ ${ }^{a}$ Fuels and Energy Technology Institute, Curtin University of Technology, Perth, WA 6845, Australia. \\ Fax: +61-8-9266 1138; E-mail: dehua.dong@curtin.edu.au \\ ${ }^{b}$ Department of Energy Materials, School of Materials Science and Engineering, Hefei University of \\ Technology, No. 193 Tunxi Road, Hefei, Anhui 230009, China.
}

${ }^{c}$ Key Lab of Design \& Assembly of Functional Nanostructure, Fujian Institute of Research on the Structure of Matter, Chinese Academy of Sciences, 155 Yangqiao Road, Fuzhou 350002, China.

\begin{abstract}
Microchanneled ceramic membranes have demonstrated superior performance in oxygen separation from air over conventional membranes. In this study, the contributions of the microchannel structure to the superior performance were investigated. Compared with supported membranes, the microchanneled membranes provide fast pathways within the channels for gas diffusion as compared to the tortuous interconnection of pore channels in the supported membranes. The walls of the numerous channels provide a large surface for facilitating oxygen dissociation, which was confirmed by varying the channel wall surface using mesh templates with different aperture sizes. In summary, the microchannel structure facilitates gas diffusion and provides a large membrane active surface, resulting in high performance in oxygen separation.
\end{abstract}

\section{Introduction}


Ceramic membranes for oxygen separation from air have attracted significant attention because the ceramic membranes operate via oxygen ion diffusion through dense solids and show theoretically $100 \%$ selectivity of oxygen separation. It is also considered as a cheaper technology than cryogenic separation, especially when the oxygen separation using ceramic membranes at high temperatures is coupled with high temperature reactions, such as natural gas conversion, coal gasification and oxyfuel combustion [1-5].

Two major issues have hindered the commercialisation of oxygen permeation membranes: low oxygen permeation rate and poor membrane material stability. Some materials with high intrinsic oxygen ion transfer rates show poor stability. For example, $\mathrm{Ba}_{0.5} \mathrm{Sr}_{0.5} \mathrm{Co}_{0.8} \mathrm{Fe}_{0.2} \mathrm{O}_{3-\delta}$ and $\mathrm{SrCo}_{0.8} \mathrm{Fe}_{0.2} \mathrm{O}_{3-\delta}$ membranes could produce an oxygen flux above $5 \mathrm{ml} \mathrm{cm}{ }^{-2} \mathrm{~min}^{-1}$ at $900{ }^{\circ} \mathrm{C}$, but they are unstable in the presence of $\mathrm{CO}_{2}$ or reducing atmosphere due to chemical decomposition [6-8]. Many materials having strong chemical toughness exhibit low oxygen permeation rates; a typical example is the dual phase membrane of $40 \mathrm{wt} \% \mathrm{NiFe}_{2} \mathrm{O}_{4}$ and 60 wt $\% \mathrm{Ce}_{0.9} \mathrm{Gd}_{0.1} \mathrm{O}_{2-\delta}[9]$.

One approach to overcoming these issues is to develop new membrane geometries and microstructures that can bring significant improvements in oxygen permeation because the membrane geometry and microstructure are not limited by materials. Hence, the materials with good chemical stability can be used to improve the oxygen permeation by modifying membrane geometry and microstructure. In our previous work, microchanneled membranes were fabricated using a mesh-templating phase inversion process [10]. With a superior structure to conventional supported membranes, the microchanneled membranes possess well-organized microchannels sitting on a thin dense layer with the other end open on the other side of the membranes. The membranes were formed by a one-step phase inversion process, and the integrated structure has high resistance to thermal shock. This 
microchanneled membrane produced high oxygen fluxes. For example, $\mathrm{La}_{0.6} \mathrm{Sr}_{0.4} \mathrm{Co}_{0.2} \mathrm{Fe}_{0.8} \mathrm{O}_{3-}$ $\delta$ microchanneled membranes achieved oxygen fluxes of $3.2 \mathrm{ml} \mathrm{cm}^{-2} \mathrm{~min}^{-1}$ at $1000{ }^{\circ} \mathrm{C}$ and $2.45 \mathrm{ml} \mathrm{cm}^{-2} \mathrm{~min}^{-1}$ at $950{ }^{\circ} \mathrm{C}$. In addition, applying Pt catalyst on the channel wall surface effectively improved the oxygen fluxes [11]. However, it is not clear precisely how the microchanneled structure contributes to the high oxygen fluxes.

In this study, we designed experiments to explore the advantages of the microchannel structure for oxygen separation from air. Conventional supported membranes were prepared to compare gas diffusion through their tortuous pore channels with that through the straight channels within the microchanneled membranes. The contribution of the channel walls for surface reaction (oxygen dissociation) is also investigated by varying the channel size using different mesh templates.

\section{Experimental}

\subsection{Fabrication of microchanneled membranes}

The fabrication process of microchanneled membranes is shown in described in Fig. 1a. It starts with slurry preparation using a planetary ball mill (MTI corporation, USA) by mixing $30 \mathrm{~g}$ of $\mathrm{La}_{0.6} \mathrm{Sr}_{0.4} \mathrm{Co}_{0.2} \mathrm{Fe}_{0.8} \mathrm{O}_{3-\delta}$ (LSCF, Fuel Cell Materials, Ohio, USA), $1.87 \mathrm{~g}$ of polyethersulfone (PESF, Radel-A300), 0.21 of g polyvinylpyrrolidone (PVP, MW=40000) and $11 \mathrm{~g}$ of 1-methyl-2-pyrrolidinone (NMP, Sigma-Aldrich, Australia) at a speed of 300 RPM for 48 hours to form a homogeneous slurry. Then, the degassed slurry was cast into a mould and then a piece of stainless steel mesh (SEFAR, Australia) was immersed just below the surface of the slurry. After phase inversion for 30 mins induced by water on the top of the slurry, the mesh was lifted up to remove the skin layer. The formed membrane green body 
was rinsed with water several times to remove the solvent residue and dried at $105{ }^{\circ} \mathrm{C}$. The prepared green bodies of the microchanneled membranes were then sintered at $1300{ }^{\circ} \mathrm{C}$ in a box furnace (ModuTemp Pty. Ltd., Australia) for 5 hours to densify the separation layer. The membranes with different channel diameters were prepared using meshes with different aperture sizes.

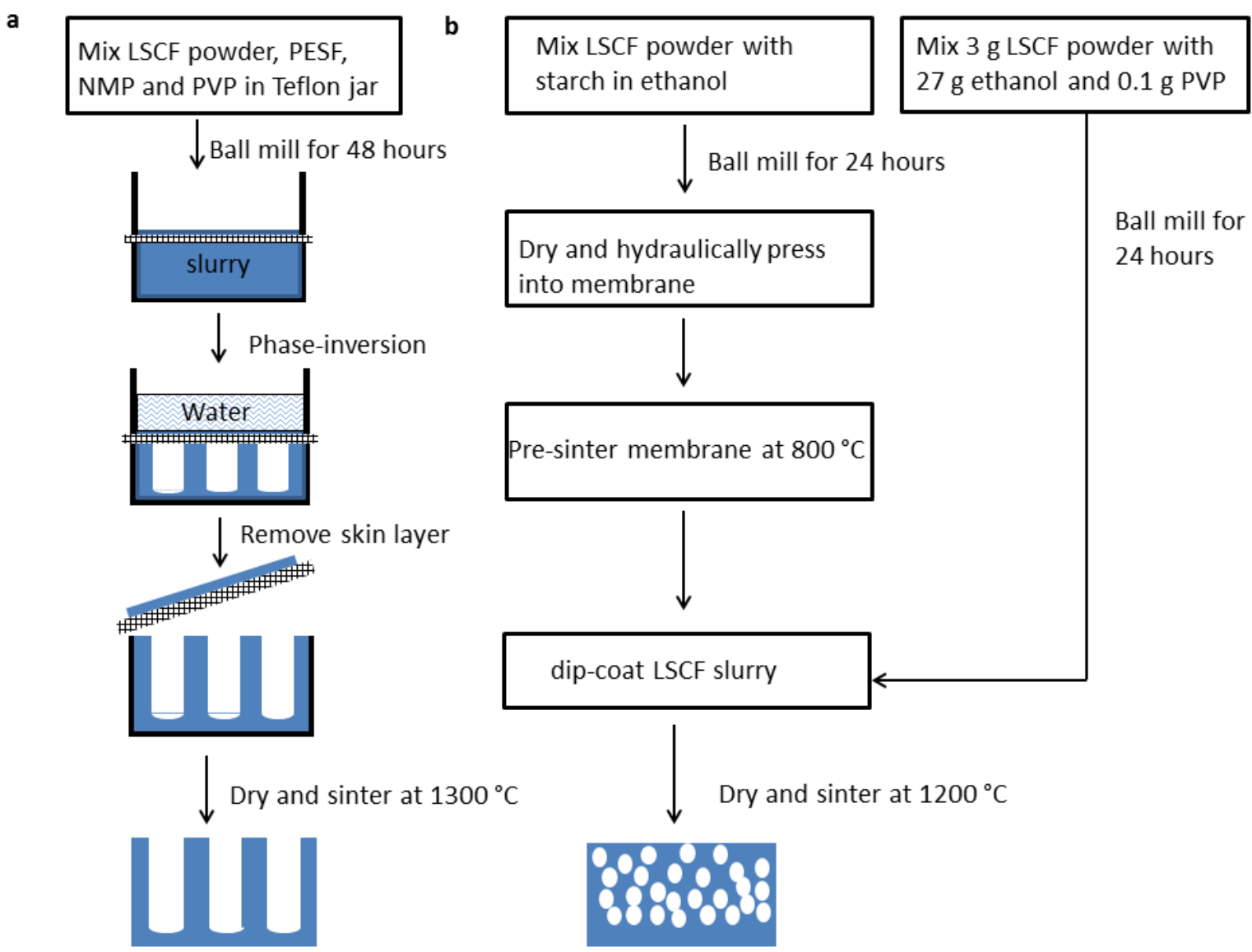

Fig. 1. Schematic representations of fabrication processes for microchanneled membrane (a) and supported membrane (b).

\subsection{Fabrication of dense and supported membranes}

Dense membranes were fabricated using a hydraulic press, followed by sintering at $1300{ }^{\circ} \mathrm{C}$. The membrane thickness was controlled by the amount of powder used to form the 
membrane, and thickness of about $1.4 \mathrm{~mm}$ was produced. The supported membrane was made by press forming a porous support and subsequently dip-coating a separation layer. The fabrication process is shown in Fig. 1b. Firstly, Pore former potato starch (Sigma-Aldrich, Australia) and LSCF powder were mixed in the mass ratio of 1:4 in ethanol using a ball mill at a speed of 400 RPM for 24 hours [12]. After drying, the mixed power was hydraulically pressed at a pressure of $300 \mathrm{MPa}$ to form the green body of the porous support. The green body was pre-sintered in a box furnace at $800{ }^{\circ} \mathrm{C}$ for 2 hours. The slurry for dip-coating was prepared by mixing $3 \mathrm{~g}$ of LSCF powder, $27 \mathrm{~g}$ of ethanol and $0.1 \mathrm{~g}$ of PVP using a ball mill at the speed of 400 RPM for 24 hours. The pre-sintered porous support was dipped into the slurry and then dried. The process was repeated for several times to achieve the desired dense layer thickness. Finally, the membrane was sintered at $1200{ }^{\circ} \mathrm{C}$ in the box furnace for 5 hours to densify the coating layer.

\subsection{Membrane characterisation and testing}

The membrane microstructure was observed by scanning electron microscopy (SEM, Zeiss Evo 40XVP). Membrane porosities were measured using the Archimedes method. In this study, the microchannel side of the microchanneled membranes and the porous side of the supported membranes were faced to the feed gas in all experiments because this test configuration can achieve higher oxygen permeation rates compared to the arrangement where the dense sides were faced to the feed gas $[10,13]$. The gas flow rates were controlled by mass flow controllers (MFC, AALBORG). Oxygen concentration in the sweep gas was measured by online gas chromatography (GC, GC-2014, Shimadzu) with a packed column of molecular sieve $5 \AA \AA$. The oxygen permeation fluxes were calculated using the following equation: 


$$
J_{O_{2}}=\frac{\mathrm{F}_{S}\left(C_{O_{2}}-C_{N_{2}}(21 / 79)\right)}{\mathrm{A}}
$$

where $J_{O_{2}}$ is the oxygen permeation flux of the membranes, $\mathrm{F}_{S}$ is the flow rate of the sweep gas, $C_{\mathrm{O}_{2}}$ is the oxygen concentration in sweep gas, $\mathrm{C}_{\mathrm{N}_{2}}$ is the concentration of nitrogen leaking into the sweep gas; A is the effective area of the membranes. All testing membranes were 14$15 \mathrm{~mm}$ in diameter with an active area of about $0.7 \mathrm{~cm}^{2}$.

\section{Results and discussion}

\subsection{Gas diffusion through microchannels}

Supported membranes with a thin dense layer on porous supports have been developed to shorten oxygen ion diffusion distances in dense membranes, and this membrane configuration has been employed to achieve high oxygen fluxes $[14,15]$. Considering that the supported membranes and the microchanneled membranes have a similar dense layer through which oxygen ions diffuse, it is feasible to compare the gas diffusion through the conventional porous supports of supported membranes and the channels of the microchanneled membranes by measuring the oxygen permeation rates. The SEM images of a microchanneled membrane and a supported membrane are shown in Fig. 2. The dense layer of the porous supported membrane is about $150 \mu \mathrm{m}$ thick. The distance from channel bottom to membrane dense surface varies from 80 to $200 \mu \mathrm{m}$, and the average oxygen ion diffusion distance is therefore about $140 \mu \mathrm{m}$. The similar oxygen ion diffusion distance makes it valid to compare gas diffusion within two different porous structures. Porous structure and microchannel structure are shown in Fig. 2c and d. The pores in the supported membrane are around 5-10 $\mu \mathrm{m}$, and the diameters of the microchannels are about $30 \mu \mathrm{m}$. The channel walls are dense. The 
porosity of the microchanneled membrane is $23 \%$, which is lower than that of the supported membrane (36\%).

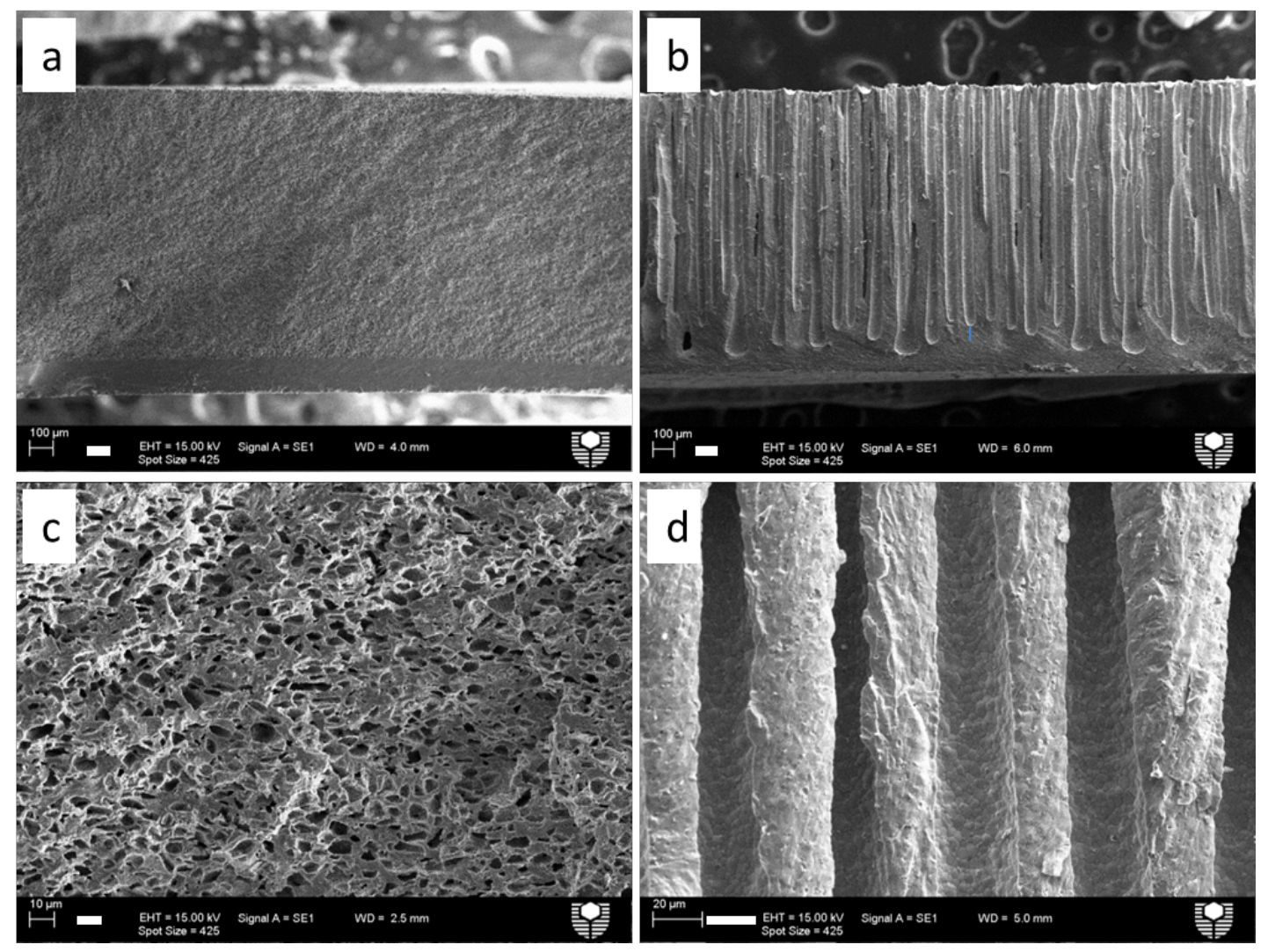

Fig. 2. SEM images of the cross sections of the supported membrane (a and c) and the microchanneled membrane (b and d) at low (a and b) and high (c and d) magnifications. The scale bars are $100 \mu \mathrm{m}$ in a and b, $10 \mu \mathrm{m}$ in $\mathrm{c}$ and $20 \mu \mathrm{m}$ in $\mathrm{d}$. The microchanneled membrane was templated by a mesh with an aperture size of $70 \mu \mathrm{m}$. 

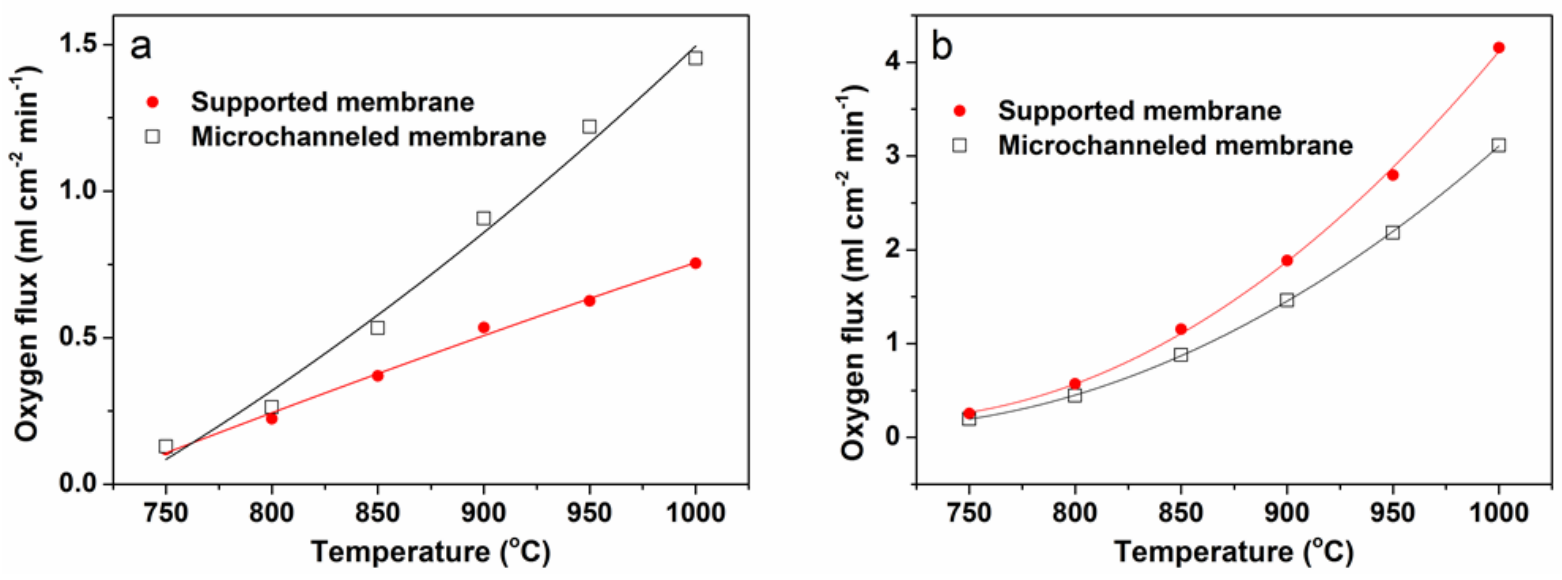

Fig. 3. Temperature dependence of the oxygen fluxes of the microchanneled membrane and the supported membrane using air (a) and pure oxygen (b) as a feed gas, respectively. The flow rates of the feeding gases were $20 \mathrm{ml} \mathrm{min}^{-1}$, and the sweep gas flow rate was maintained at $100 \mathrm{ml} \mathrm{min}^{-1}$.

As shown in Fig. 3a, the oxygen flux of the microchanneled membrane is higher than that of the supported membrane. As the operating temperature was increased, the difference in the oxygen flux increased. For example, the oxygen fluxes of the microchanneled membrane and the supported membrane are 0.13 and $0.12 \mathrm{ml} \mathrm{cm}^{-2} \mathrm{~min}^{-1}$ at $750{ }^{\circ} \mathrm{C}$, and 1.5 and $0.76 \mathrm{ml} \mathrm{cm}^{-2}$ $\min ^{-1}$ at $1000{ }^{\circ} \mathrm{C}$, respectively. The difference enlargement might be caused by the difference of gas diffusion resistance through the two membranes. That is to say that the build up of concentration of nitrogen within the pores/channels, and its back diffusion out of the membrane will hinder the influx of oxygen in fresh air. Cao et al. reported the presence of gas diffusion resistance/concentration polarization within the porous supports of membranes at high oxygen fluxes [16]. The gas diffusion resistance becomes obvious as the oxygen flux is increased with operating temperature, resulting in the enlarged difference in oxygen flux between the two types of membranes. 
To investigate this interpretation, the two membranes were tested using pure oxygen as a feed gas to eliminate gas diffusion resistance. As the kinetics of oxygen surface exchange increase with the oxygen partial pressure regardless of membrane structure, it is valid to compare by feeding oxygen. Without nitrogen dilution, there would be no oxygen concentration gradient and therefore no gas diffusion resistance. As shown in Fig. 3b, with oxygen as a feed gas, the supported membrane showed a higher oxygen flux than the microchanneled membrane; for example, the fluxes were 4.2 and $3.1 \mathrm{ml} \mathrm{cm}^{-2} \mathrm{~min}^{-1}$ for the supported membrane and the microchanneled membrane at $1000{ }^{\circ} \mathrm{C}$, respectively. The higher oxygen flux of the supported membrane is attributed to its higher surface area within the porous support compared to that of the microchannel walls. Overall, compared with conventional supported membranes, microchanneled membranes provide straight channels as gas diffusion pathways instead of the tortuous pore channels within the porous support, greatly facilitating gas diffusion and therefore leading to high oxygen fluxes when the feed gas is air [17].

To further investigate the gas diffusion resistance, the activation energy of the oxygen permeation through the supported membranes and microchanneled membranes were measured. Compared with conventional dense membranes, the supported membranes and microchanneled membranes involve gas diffusion through pores/channels during the oxygen permeation. It is known that the activation energy of the gas diffusion (less than $20 \mathrm{~kJ} \mathrm{~mol}^{-1}$ ) is much smaller than those of ionic bulk diffusion (about $115 \mathrm{~kJ} \mathrm{~mol}^{-1}$ ) and surface reaction (about $191 \mathrm{~kJ} \mathrm{~mol}^{-1}$ ) [18, 19]. Therefore, it is possible to identify the presence of gas diffusion resistance on the basis of activation energy measurements. 

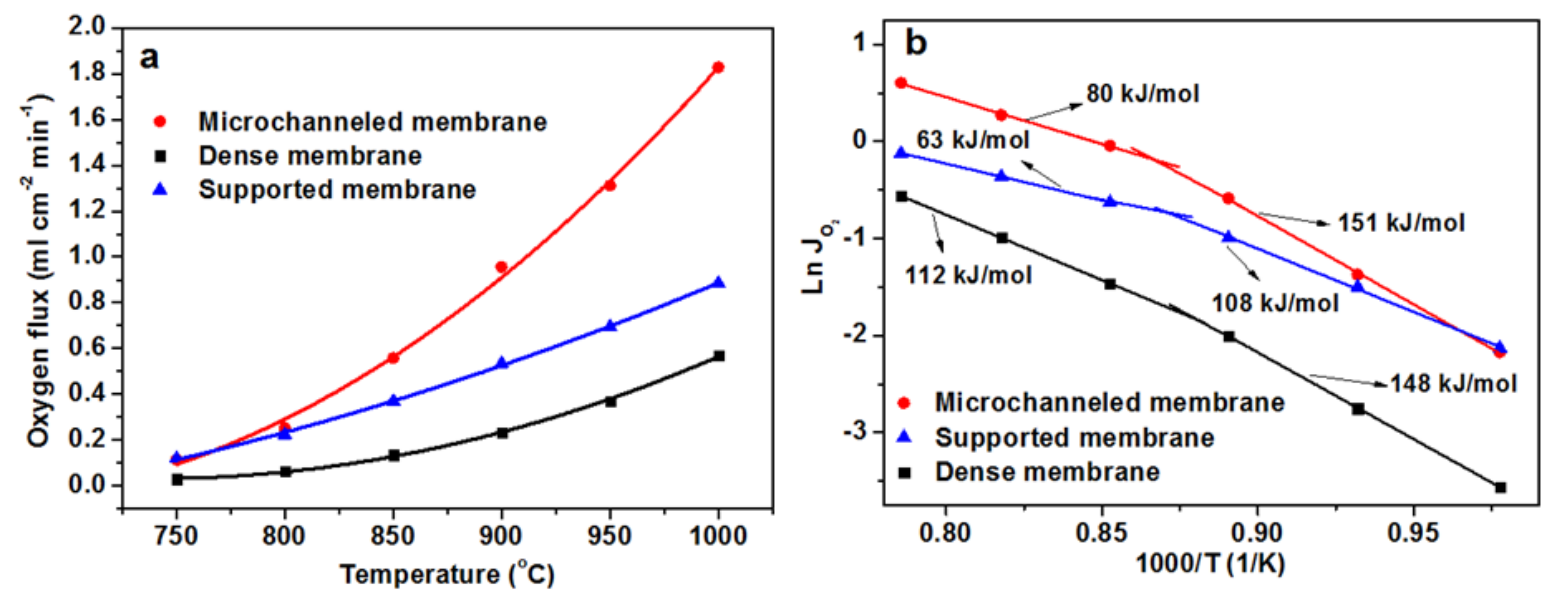

Fig. 4 a. Oxygen fluxes of the microchanneled membrane templated by a mesh with an aperture size of $70 \mu \mathrm{m}$, a supported membrane and a dense membrane. The oxygen partial pressure difference on two sides of the membranes was maintained at $0.21 / 0.004 \mathrm{~atm}$ and the air flow rate was $100 \mathrm{ml} \mathrm{min}^{-1}$. b. Arrhenius plots and activation energies calculated according to Fig. 4a.

Fig. 4a shows the oxygen permeation through a microchanneled membrane, a supported membrane and a dense membrane with the membrane thickness of about $1.4 \mathrm{~mm}$ with an oxygen partial pressure gradient of 0.21/0.004 atm, and the corresponding Arrhenius curves are plotted in Fig.4b. As there is no gas diffusion involved in the oxygen permeation through dense membranes, the activation energy of the oxygen permeation through a dense membrane was also tested as a reference. Within the high temperature range (900-1000 ${ }^{\circ} \mathrm{C}$ ), the activation energy of the dense membrane is close to that of oxygen ionic diffusion (115 kJ $\mathrm{mol}^{-1}$ ) in LSCF membranes, which indicates that the oxygen permeation of the dense membranes is rate-limited by oxygen ion diffusion in the solid phase. However, the activation energy was increased to around $150 \mathrm{~kJ} \mathrm{~mol}^{-1}$ in the low temperature range $\left(750-900{ }^{\circ} \mathrm{C}\right)$; these values are located between those of the activation energy of oxygen ionic diffusion and 
surface reactions (191 kJ mol${ }^{-1}$ ), suggesting that the oxygen permeation through the dense membranes was jointly rate-limited by oxygen ionic diffusion and surface reactions.

As the activation energy of gas diffusion is less than $20 \mathrm{~kJ} \mathrm{~mol}^{-1}$, the existence of gas diffusion resistance in the oxygen permeation will decrease the overallactivation energy of the oxygen permeation. In the high temperature range, the gas diffusion resistance is more likely to occur because of the large oxygen fluxes. From Fig. 4b, the activation energies for the oxygen permeation of supported and microchanneled membranes are 63 and $80 \mathrm{~kJ} \mathrm{~mol}^{-}$ ${ }^{1}$ respectively, which are much smaller than the activation energy of ionic bulk diffusion (112 $\mathrm{kJ} \mathrm{mol}^{-1}$ ). This suggests the existence of gas diffusion resistance in the oxygen permeation through both the membranes. Moreover, compared with the supported membrane, the microchanneled membrane showed a higher activation energy, indicating less gas diffusion resistance and hence improved gas diffusion through straight microchannels. The gas diffusion resistances can also be observed in the low temperature range on the basis of activation energies, although the gas diffusion resistance is not as serious as it is in the high temperature range due to the lower oxygen fluxes. However, the activation energies of oxygen permeation though the dense membrane and microchanneled membrane at low temperature are similar (151 vs $148 \mathrm{~kJ} \mathrm{~mol}^{-1}$ ). Compared with the dense membrane, the microchanneled membrane has shorter ionic diffusion distance, and therefore the oxygen permeation is more controlled by surface reaction, which increases the overall activation energy. At the same time, the existence of gas diffusion resistance decreases the activation energy. The two effects cancel each other out, leading to no net change in activation energies. For the supported membrane, gas diffusion resistance contributed more to the permeation process, resulting in the lower activation energy of $108 \mathrm{~kJ} \mathrm{~mol}^{-1}$.

\subsection{The effect of membrane surface within channels}




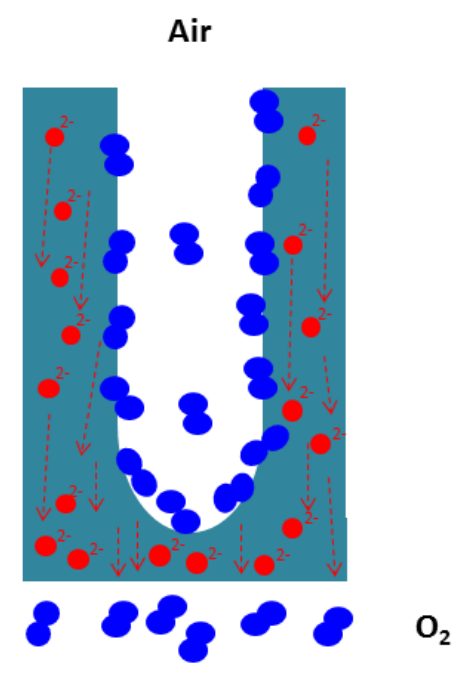

Fig. 5. Schematic illustration of oxygen permeation paths through a channel wall surface

Apart from facilitating gas diffusion through membranes, the microchannel structure potentially increases the membrane active surface area as the surface of numerous channel walls can conduct oxygen dissociation, as illustrated in Fig. 5. The channel wall contribution decreases with the distance from the channel bottom. To identify the contribution, membranes with different channel surfaces were prepared by using mesh templates with different aperture sizes.

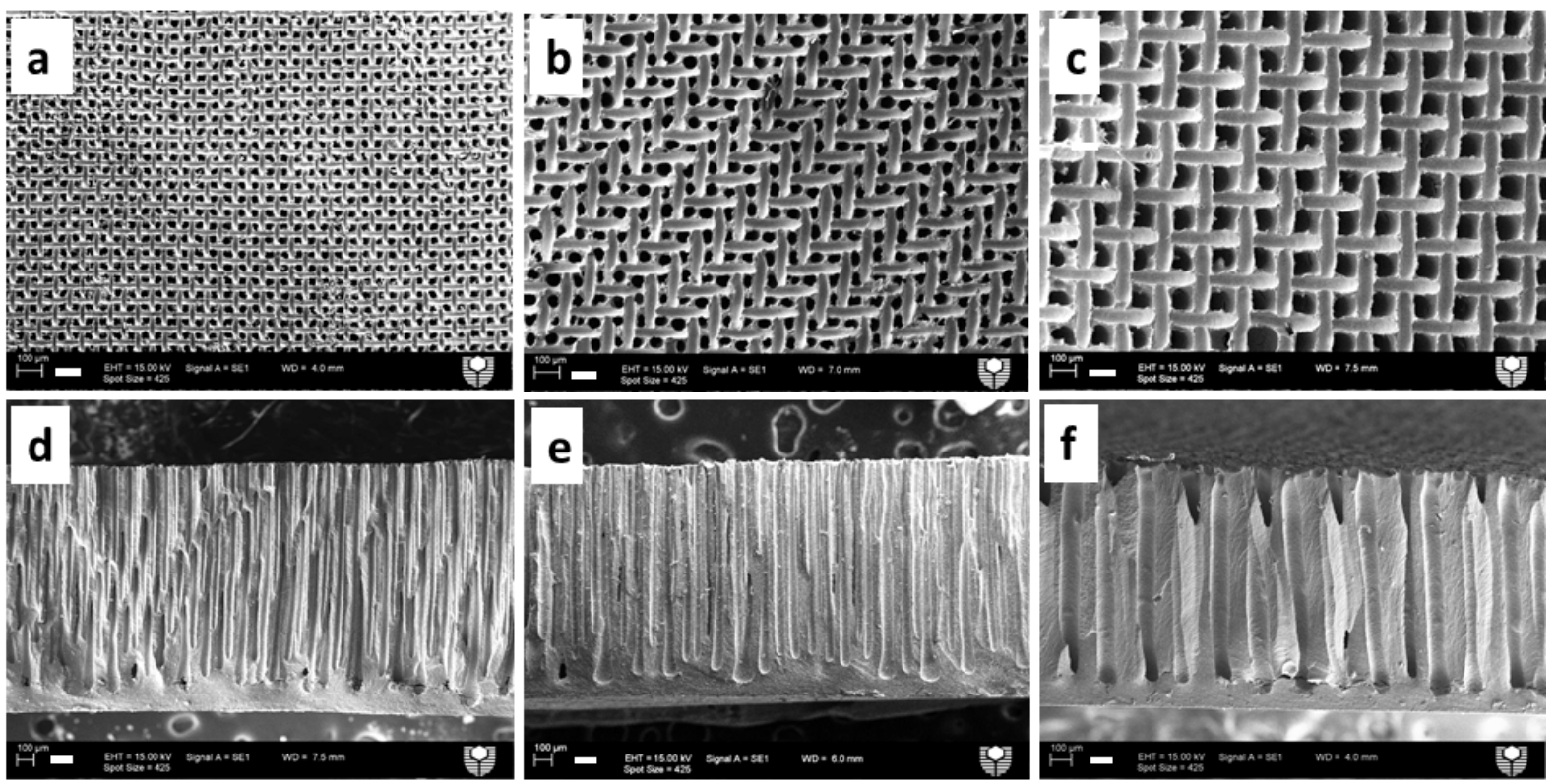


Fig. 6. SEM images of surfaces ( $a, b$ and $c$ ) and cross sections (d, e and f) of the membranes templated by the meshes with the aperture sizes of $55 \mu \mathrm{m}$ ( $\mathrm{a}$ and $\mathrm{d}$ ), 70 ( $\mathrm{b}$ and d) and $150 \mu \mathrm{m}$ (c and f). The scale bars are $100 \mu \mathrm{m}$.

The mesh-templating phase inversion process can control the diameter of the channels by using meshes with different aperture sizes, which results in different channel wall surface areas. Narrow channels provide a large wall surface area, per unit area of membrane. Three steel meshes with the aperture sizes of $55 \mu \mathrm{m}, 70 \mu \mathrm{m}$ and $150 \mu \mathrm{m}$ were used. As shown in Fig. 6, uniform channels were templated by the mesh apertures, and the channel diameter increases with mesh aperture size. Although the mesh aperture is square, the cross section of the templated channels is circular due to the surface tension of the slurry. For the mesh aperture size of $150 \mu \mathrm{m}$, the initial cross section of the templated channels is square. However, it becomes circular as the channels grow away from the mesh. The cross sections of the membranes showed that all the membranes have a similar dense separation layer of about 150 $\mu \mathrm{m}$ in thicknesses, which makes it feasible to compare the channel wall contribution by testing oxygen permeations.

The channel diameters were measured from the SEM images. The channel diameter distributions are shown in the Fig. 7, and it is noted the microchannel sizes tend to be more uniform when they are templated using meshes with smaller aperture sizes. The mean pore diameters were calculated to be $25 \mu \mathrm{m}, 40 \mu \mathrm{m}$ and $87 \mu \mathrm{m}$ for the microchanneled membranes templated by the meshes with the aperture sizes of $55 \mu \mathrm{m}, 70 \mu \mathrm{m}$ and $150 \mu \mathrm{m}$, respectively.

Assuming the channels have perfect cylindrical shapes and have the same length, the theoretical surface area of channel wall can be calculated using the following equation: $S=\pi d l N$ 
where $\mathrm{N}$ is the channel numbers in $1 \mathrm{~cm}^{2}$ of membrane area

$\mathrm{N}$ is calculated by dividing membrane pore/void volume with single channel volume:

$N=\frac{4 \mathrm{PL}}{\pi l d^{2}}$

where $\mathrm{P}$ is porosity; $\mathrm{L}$ is membrane thickness; $\mathrm{l}$ is channel length; $\mathrm{d}$ is channel diameter.
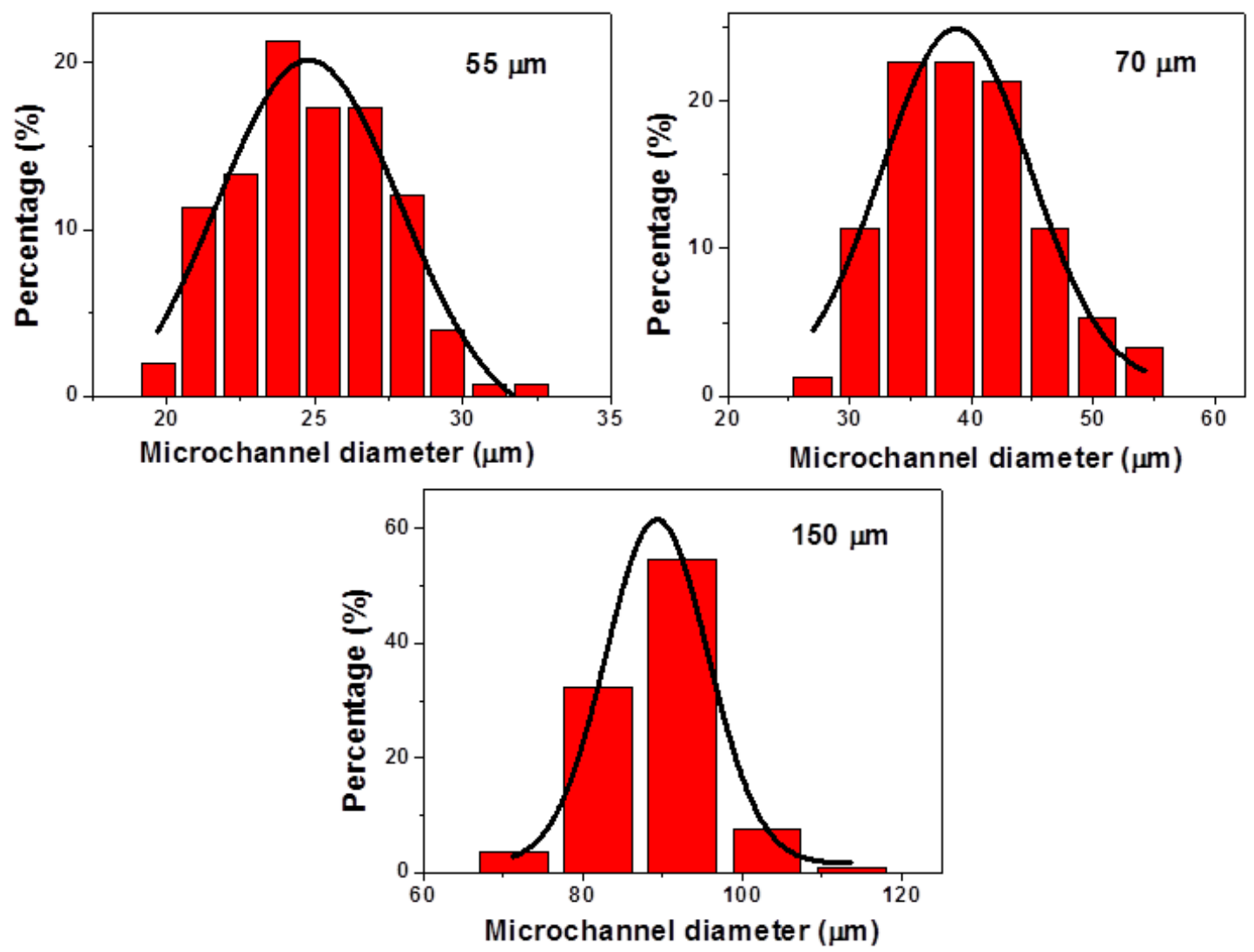

Fig. 7. Channel diameter distribution of the membranes templated by the meshes with different aperture sizes. 


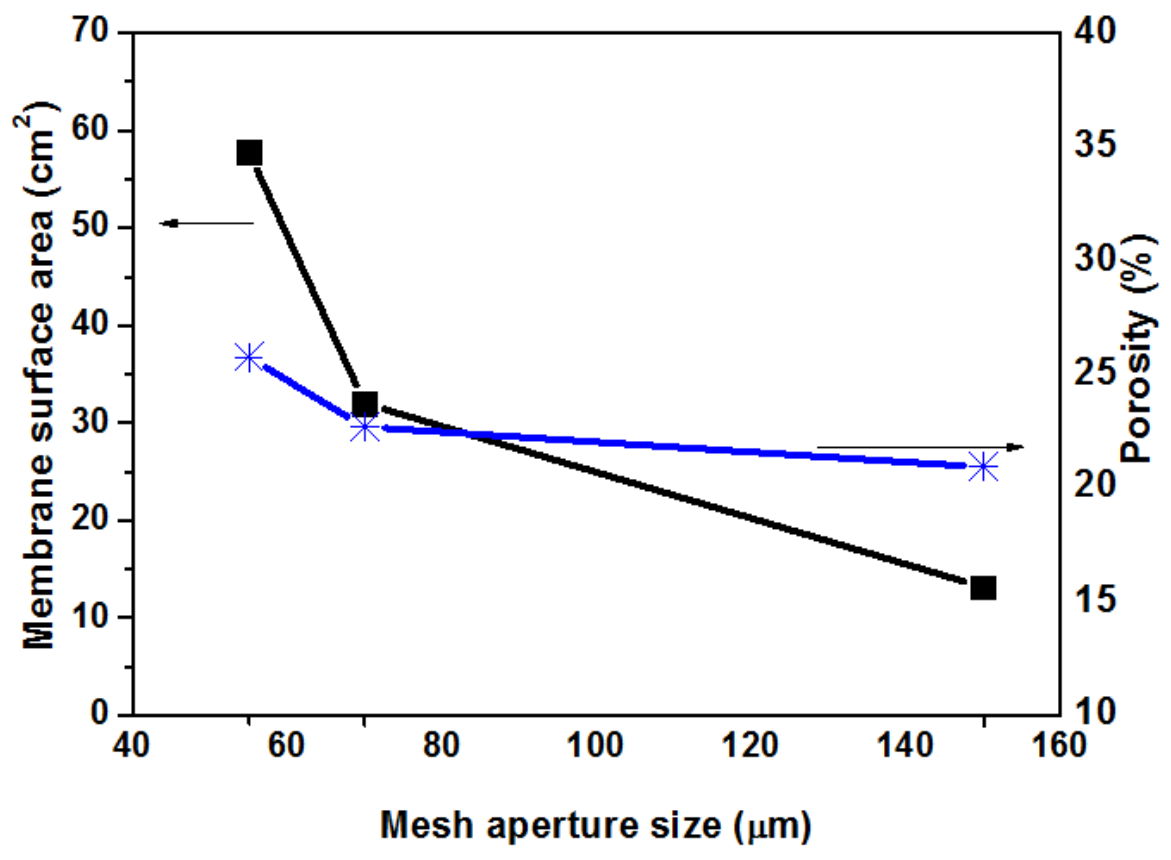

Fig. 8. Theoretical membrane surface areas for the microchanneled membranes templated by the meshes with the aperture sizes of $55 \mu \mathrm{m}, 70 \mu \mathrm{m}$ and $150 \mu \mathrm{m}$.

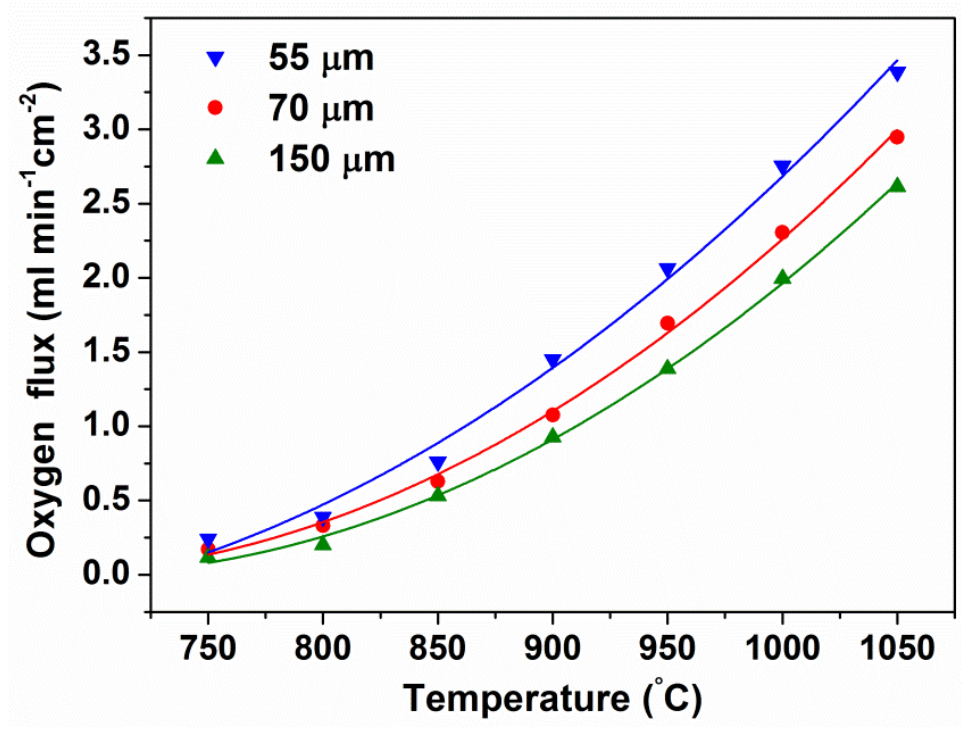

Fig. 9. Oxygen fluxes of the membranes templated by the meshes with different aperture sizes 55, 70 and $150 \mu \mathrm{m}$. Both sweep gas rate and air flow rate were $270 \mathrm{ml} \mathrm{min}^{-1}$.

Fig.8 demonstrates that the membrane surface area varies with mesh aperture sizes. The membrane surface area and porosities both decrease with increasing channel size. The 
microchanneled membrane templated by the mesh with an aperture size of $55 \mu \mathrm{m}$ has a surface area of $58 \mathrm{~cm}^{2}$, while it is only $13 \mathrm{~cm}^{2}$ for the microchanneled membrane templated by the mesh with an aperture size of $150 \mu \mathrm{m}$. The membrane templated by the mesh with an aperture size of $55 \mu \mathrm{m}$ has the largest channel wall surface area compared with other membranes and should therefore produce the highest oxygen flux; this is confirmed by the experimental results shown in Fig. 9. At $1050^{\circ} \mathrm{C}$, the membrane made by the mesh with an aperture size of $55 \mu \mathrm{m}$ produced an oxygen flux of $3.4 \mathrm{ml} \mathrm{cm}^{-2} \mathrm{~min}^{-1}$, which is higher than that of the other membranes. For example, the membrane made by a mesh with an aperture size of $150 \mu \mathrm{m}$ only achieved an oxygen flux of $2.6 \mathrm{ml} \mathrm{cm}^{-2} \mathrm{~min}^{-1}$. These resultsconfirm that the channel wall surface contributes to the oxygen permeation and higher oxygen permeation rates can be achieved by reducing the channel diameter.

\section{Conclusions}

The microchanneled membranes have produced higher oxygen fluxes during oxygen separation from air compared with conventional dense membranes and supported membranes. The superior performance is attributed to the microchannel structure: straight channels provide fast pathway for oxygen molecular diffusion to the thin separation layer compared with the tortuous pore channels within porous supports; numerous microchannels also offer large active membrane surface for oxygen dissociation, which is confirmed by comparing the performance of membranes with different channel diameters. The advantages of the microchannel structure can potentially be applied in other applications such as solid oxide fuel cells and batteries [20-22].

\section{Acknowledgements}


This work was financially supported by Australian Research Council Discovery Project

(DP120103317), the government of Western Australia via the Centre for Research into

Energy for Sustainable Transport (CREST) and Curtin University of Technology through the

Curtin Research Fellowship scheme. We also thank for the Curtin Centre for Materials

Research for help with SEM imaging.

\section{References}

[1] U. Balachandran, J. Dusek, R.L. Mieville, R. Poeppel, M. Kleefisch, S. Pei, T. Kobylinski, C. Udovich, A. Bose, Dense ceramic membranes for partial oxidation of methane to syngas, Appl. Catal. A-Gen. 133 (1995) 19-29.

[2] U. Balachandran, J. Dusek, P. Maiya, B. Ma, R. Mieville, M. Kleefisch, C. Udovich, Ceramic membrane reactor for converting methane to syngas, Catal. Today 36 (1997) 265-272.

[3] A. Leo, S. Liu, J.C.D.d. Costa, Development of mixed conducting membranes for clean coal energy delivery, Int. J. Greenh. Gas Con. 3 (2009) 357-367.

[4] S. Smart, C. Lin, L. Ding, K. Thambimuthu, J.D. Da Costa, Ceramic membranes for gas processing in coal gasification, Energ. Environ. Sci. 3 (2010) 268-278.

[5] H. Stadler, F. Beggel, M. Habermehl, B. Persigehl, R. Kneer, M. Modigell, P. Jeschke, Oxyfuel coal combustion by efficient integration of oxygen transport membranes, Int. J. Greenh. Gas Con. 5 (2011) 7-15.

[6] B.J. Mitchell, R.C. Rogan, J.W. Richardson Jr, B. Ma, U. Balachandran, Stability of the cubic perovskite SrFe0.8Co0.2O3- $\delta$, Solid State Ionics 146 (2002) 313-321.

[7] S. McIntosh, J.F. Vente, W.G. Haije, D.H.A. Blank, H.J.M. Bouwmeester, Phase stability and oxygen non-stoichiometry of SrCo0.8Fe0.2O3- $\delta$ measured by in situ neutron diffraction, Solid State Ionics 177 (2006) 833-842.

[8] M. Arnold, T.M. Gesing, J. Martynczuk, A. Feldhoff, Correlation of the formation and the decomposition process of the BSCF perovskite at intermediate temperatures, Chem. Mater. 20 (2008) 5851-5858.

[9] H. Luo, K. Efimov, H. Jiang, A. Feldhoff, H. Wang, J. Caro, CO2-Stable and Cobalt-Free Dual-Phase Membrane for Oxygen Separation, Angew. Chem. Int. Ed. 50 (2011) 759-763.

[10] X. Shao, D. Dong, G. Parkinson, C.-Z. Li, A microchanneled ceramic membrane for highly efficient oxygen separation, J. Mater. Chem. A 1 (2013) 9641-9644.

[11] X. Shao, D.H. Dong, G. Parkinson, C.Z. Li, Improvement of oxygen permeation through microchanneled ceramic membranes, J. Membr. Sci. 454 (2014) 444-450.

[12] G. Etchegoyen, T. Chartier, P. Del-Gallo, An architectural approach to the oxygen permeability of a La0.6Sr0.4Fe0.9Ga0.103- $\delta$ perovskite membrane, J. Eur. Ceram. Soc. 26 (2006) 2807-2815.

[13] X. Chang, C. Zhang, X. Dong, C. Yang, W. Jin, N. Xu, Experimental and modeling study of oxygen permeation modes for asymmetric mixed-conducting membranes, J. Membr. Sci. 322 (2008) 429435.

[14] S. Baumann, J.M. Serra, M.P. Lobera, S. Escolástico, F. Schulze-Küppers, W.A. Meulenberg, Ultrahigh oxygen permeation flux through supported $\mathrm{Ba} 0.5 \mathrm{Sr} 0.5 \mathrm{Co0} .8 \mathrm{Fe} 0.2 \mathrm{O}-\delta$ membranes, J. Membr. Sci. 377 (2011) 198-205.

[15] S. Baumann, W.A. Meulenberg, H.P. Buchkremer, Manufacturing strategies for asymmetric ceramic membranes for efficient separation of oxygen from air, J. Eur. Ceram. Soc. 33 (2013) 12511261. 
[16] Z. Cao, X. Zhu, W. Li, B. Xu, L. Yang, W. Yang, Asymmetric dual-phase membranes prepared via tape-casting and co-lamination for oxygen permeation, Mater. Lett. 147 (2015) 88-91.

[17] R.E. Williford, L.A. Chick, G.D. Maupin, S.P. Simner, J.W. Stevenson, Diffusion Limitations in the Porous Anodes of SOFCs, J. Electrochem. Soc. 150 (2003) A1067-A1072.

[18] A.B. Shelekhin, A.G. Dixon, Y.H. Ma, Theory of gas diffusion and permeation in inorganic molecular-sieve membranes, AIChE J. 41 (1995) 58-67.

[19] S.J. Xu, W.J. Thomson, Oxygen permeation rates through ion-conducting perovskite membranes, Chem. Eng. Sci. 54 (1999) 3839-3850.

[20] C.-J. Bae, C.K. Erdonmez, J.W. Halloran, Y.-M. Chiang, Design of Battery Electrodes with DualScale Porosity to Minimize Tortuosity and Maximize Performance, Adv. Mater. 25 (2013) 1254-1258. [21] X. Lu, G. Coffey, K. Meinhardt, V. Sprenkle, Z. Yang, J.P. Lemmon, High Power Planar SodiumNickel Chloride Battery, ECS Transactions 28 (2010) 7-13.

[22] D. Dong, X. Shao, K. Xie, X. Hu, G. Parkinson, C.-Z. Li, Microchanneled anode supports of solid oxide fuel cells, Electrochem. Commun. 42 (2014) 64-67. 